\title{
A heart within a heart
}

\author{
Christopher Labos MD CM, Shadi Akhtari MD
}

A 66-year-old man with a recent diagnosis of oculopharyngeal muscular dystrophy presented to a medical clinic with a complaint of progressive fatigue. An electrocardiogram performed in the clinic showed inferior $Q$ waves consistent with a distant myocardial infarction. An echocardiogram was ordered to assess the patient's ejection fraction and any wall motion abnormalities. The four-chamber view of the heart demonstrated a $24 \mathrm{~mm} \times 7 \mathrm{~mm}$ mass

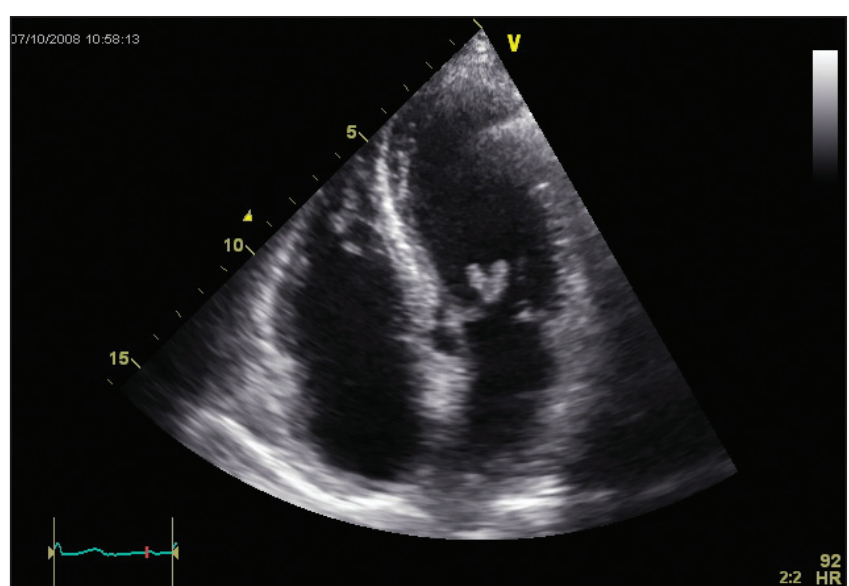

Figure 1 attached to the midportion of the atrial side of the anterior leaflet of the mitral valve. The mass had a peculiar heart shape (Figures 1 and 2). Ophthalmological examination of the fundus revealed Roth spots. Blood cultures were positive for Streptococcus mitis. The patient was diagnosed with endocarditis and started on intravenous antibiotics. He was discharged from the hospital 10 days later in good condition.

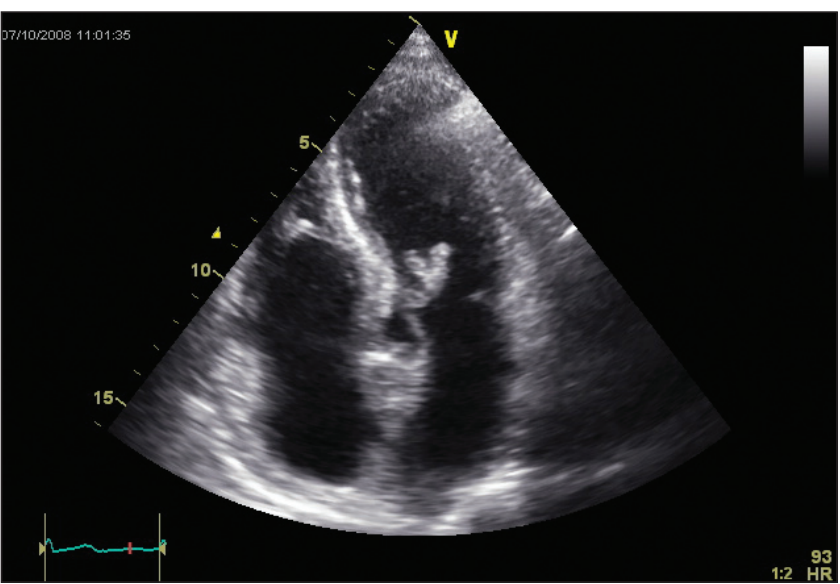

Figure 2

Department of Cardiology, McGill University Health Centre, Montreal, Quebec

Correspondence: Dr Christopher Labos, Royal Victoria Hospital, Cardiology Division, Room M4.76, McGill University Health Centre, Royal Victoria

Hospital, 687 ave des Pins Ouest, Montreal, Quebec H3A 1A1. Telephone 514-934-1934 ext 36151, fax 514-843-2318,

e-mail christopher.labos@mail.mcgill.ca

Received for publication October 31, 2009. Accepted December 2, 2009 